\title{
Abnormal Mammary Adipose Tissue Environment of Brca1 Mutant Mice Show a Persistent Deposition of Highly Vascularized Multilocular Adipocytes
}

\author{
Laundette P. Jones $^{1 *}$, Destiney Buelto ${ }^{2}$, Elaine Tago ${ }^{3}$ and Kwadwo E. Owusu-Boaitey ${ }^{4}$ \\ ${ }^{1}$ Department of Pharmacology and Experimental Therapeutics, University of Maryland, Baltimore, School of Medicine, Baltimore, MD 21201 \\ ${ }^{2}$ Department of Biology, University of North Carolina, Chapel Hill, Chapel Hill, NC 27599 \\ ${ }^{3}$ American Samoa Community College, P.O. Box 4104, Pago Pago, American Samoa 96799 \\ ${ }^{4}$ Department of Biological Sciences, University of Maryland, Baltimore County, Baltimore, MD 21250
}

\begin{abstract}
A major challenge to breast cancer research is the identification of alterations in the architecture and composition of the breast that are associated with breast cancer progression. The aim of the present investigation was to characterize the mammary adipose phenotype from Brca1 mutant mice in the expectation that this would shed light on the role of the mammary tissue environment in the early stages of breast tumorigenesis. We observed that histological sections of mammary tissue from adult Brca1 mutant mice abnormally display small, multilocular adipocytes that are reminiscent of brown adipose tissue (BAT) as compared to wildtype mice. Using a marker for BAT, the uncoupling protein 1 (UCP1), we demonstrated that these multilocular adipose regions in Brca1 mutant mice stain positive for UCP1. Transcriptionally, UCP1 mRNA levels in the Brca1 mutant mice were elevated greater than 50-fold compared to age-matched mammary glands from wildtype mice. Indeed, BAT has characteristics that are favorable for tumor growth, including high vascularity. Therefore, we also demonstrated that the multilocular brown adipose phenotype in the mammary fat pad of Brca1 mutant mice displayed regions of increased vascularity as evidenced by a significant increase in the protein expression of CD31, a marker for angiogenesis. This Brca1 mutant mouse model should provide a physiologically relevant context to determine whether brown adipose tissue can play a role in breast cancer development.
\end{abstract}

\section{Introduction}

The mammary gland undergoes a dynamic series of alterations in its architecture and morphology during normal development as well as the development of breast cancer [1-4]. Understanding which alterations in the tissue architecture accompany the development of breast cancer is likely to improve the early detection and ultimately, the treatment of patients. The mammary gland is made up of two components, the epithelial parenchyma, and the surrounding stroma which contains a variety of cell types including, the extracellular matrix, fibroblasts, resident macrophages, and adipocytes [1-4]. Breast cancer is thought to occur initially in mammary epithelial cells (MECs) [1-2]. While MECs that harbor mutations can often initiate breast tumorigenesis, studies have shown that the stromal microenvironment surrounding MECs can play a major role in tumor cell fate [5]. Remarkably, studies have shown that this tissue environment can act as both a barrier to tumorigenesis by suppressing tumor growth or it can act as a permissive environment for tumor growth promoting MECs towards transformation and a malignant state [5].

Compositionally, adipose tissue is the major contributor to the volume of the breast. Two types of adipose tissue have been distinguished histologically and functionally [6,7]. White adipose tissue (WAT) is composed of cells with a single (unilocular) large lipid droplet that is stored and used as fuel for the organism [6,7]. In contrast, brown adipose tissue (BAT) is composed of cells with numerous (multilocular) small lipid droplets with capillaries that weave through individual brown adipocytes to facilitate the burning of lipids for heat production [6,7]. In the breast, WAT is seen continuously from birth to adult, whereas BAT often has a temporal appearance [8,9]. Insights into the contribution of BAT in mammary gland and the regulation of its temporal appearance are rather limited.
Two independent studies in mice have demonstrated the presence of BAT during the early stages of mammary gland development $[8,9]$. In the mouse, multilocular brown adipocytes are detectable in the mammary fat pad from birth until about 8-10 weeks of age (near the completion of puberty) $[8,9]$. The disappearance of these multilocular cells after puberty also correlates with the loss of the uncoupling protein 1 (UCP1), an uncoupling protein found in the mitochondria of BAT $[8,9]$. Considering that high amounts of brown adipocytes are exclusively detected during the early stages of mammary gland development, one can speculate that BAT may play a specific role in mammary ductal outgrowth. Supporting this hypothesis are historical mammary gland transplantation experiments that demonstrate that interscapular BAT can be used as a matrix to regenerate a branching system of ducts from explants of rodent mammary ducts [10].

We have previously utilized a preclinical mouse model of BRCA1-associated breast cancer in several studies to determine how exogenous factors alter the onset of mammary preneoplasia and tumor

*Corresponding author: Laundette P. Jones, PhD, University of Maryland Baltimore, Bressler Bldg., Rm 4-002, 655 W. Baltimore Street, Baltimore MD 21201 USA, Tel: 410-706-7331; Fax: 410-706-0032; E-mail: ljone010@umaryland.edu

Received November 23, 2011; Accepted December 06, 2011; Published December 08, 2011

Citation: Jones LP, Buelto D, Tago E, Owusu-Boaitey KE (2011) Abnorma Mammary Adipose Tissue Environment of Brca1 Mutant Mice Show a Persistent Deposition of Highly Vascularized Multilocular Adipocytes. J Cancer Sci Ther S2. doi:10.4172/1948-5956.S2-004

Copyright: @ 2011 Jones LP, et al. This is an open-access article distributed unde the terms of the Creative Commons Attribution License, which permits unrestricted use, distribution, and reproduction in any medium, provided the original author and source are credited. 
Citation: Jones LP, Buelto D, Tago E, Owusu-Boaitey KE (2011) Abnormal Mammary Adipose Tissue Environment of Brca1 Mutant Mice Show a Persistent Deposition of Highly Vascularized Multilocular Adipocytes. J Cancer Sci Ther S2. doi:10.4172/1948-5956.S2-004

Page 2 of 6

development [11-13]. Women with inherited mutations in the Breast Cancer Susceptibility gene-1 (BRCA1) gene have an increased risk of developing breast cancer [14]. Similar to human BRCA1-mutated breast cancers, the mammary tumors developed in these mice are classified as high-grade undifferentiated adenocarcinomas, demonstrate loss of p53 function, and are ERa negative [15-18]. In the course of our studies, we observed that postpubertal Brcal mutant mice displayed an unexpected sustained mammary adipose histology of small multilocular adipocytes that was reminiscent of brown adipose tissue (BAT) as compared to wildtype mice. Notably, BAT has characteristics that are also favorable for tumor growth, including increased vascularity [6,7]. Therefore, the aim of the present investigation was to characterize the mammary adipose phenotype in the mammary gland of the Brcal mutant mice in the expectation that this would shed light on the role of the mammary tissue environment in the early stages of breast tumorigenesis.

\section{Materials and Methods}

\section{Mice and, genotyping}

Brcal conditional knockout mice with two floxed Brcal alleles $\left(\mathrm{Brcal}^{\mathrm{f} / \mathrm{f}}\right)$ carrying the mouse mammary tumor virus (MMTV)-Cre recombinase gene (Brcal $\left.{ }^{\mathrm{f} / \mathrm{f} ; \mathrm{MMTV}-\mathrm{Cre}}\right)$ were maintained on a $\mathrm{C} 57 \mathrm{Bl} / 6$ genetic background $(n=20)$ [15]. Non-transgenic C57Bl/6 mice were used as controls $(n=20)$. Mice were maintained in temperaturecontrolled and light-controlled conditions in the University of Maryland, Baltimore animal facility. All mice were maintained in accordance with institutional guidelines approved by the University of Maryland, Baltimore Animal Care and Use Committee. Water was supplied from sterile plastic water packs. A rodent diet (Harlan Teklad Global 2019; Madison, WI) was supplied ad libitum. The presence or absence of the floxed Brca1 alleles, of wild-type Brca1 alleles, and of the MMTV-Cre was identified using polymerase chain reaction (PCR) on tail DNA as described previously $[15,19]$. Fourth mammary glands were surgically removed at necropsy and processed for a whole mount analysis or formalin fixed for histology, immunohistochemistry (IHC), and western blot analysis.

\section{Mammary gland whole mounted analysis, histological studies, and immunohistochemistry}

One \#4 mammary gland from each animal was dissected and spread on a glass slide at the time of necropsy for whole mount analyses as previously described [11-13]. The other \#4 mammary gland from each mouse was fixed in $10 \%$ buffered formalin (Fisher Scientific, Pittsburgh, PA) overnight at $4^{\circ} \mathrm{C}$ and embedded in paraffin using standard techniques. Five micron sections were cut for hematoxylin and eosin (H\&E) staining and IHC detection was performed using methods described previously [11-13] using goat anti-UCP1 polyclonal antibody (sc-6528 Santa Cruz Biotechnology, Santa Cruz, CA) and rat monoclonal to CD31 (ab7388, Abcam, San Francisco, CA). Digital photographs were taken using a Nikon 50i Upright Microscope System with a high Resolution 5 Megapixel Color Digital Camera system (Nikon Instruments Inc., Melville, NY, USA).

\section{RNA isolation and QPCR}

Total RNA was isolated from mammary glands using the Qiagen Lipid RNA Easy kit (Qiagen, Valencia CA) and quantified on a NanoDrop ND-1000 Spectrophotometer. Messenger RNA (mRNA) was reverse transcribed (RT reaction) into cDNA $\left(1 \mathrm{~h}\right.$ at $37^{\circ} \mathrm{C}$,
Programmable Thermal Controller, MJ Research, Cambridge, MA). Real-time PCR probes were purchased from Roche (Roche Applied Science, Indianapolis, IN). All real-time PCR was performed using RNA samples from three separate mice. The oligonucleotide primers used were as follows: UCP1 upstream: ggcctctacgactcagtcca; UCP1 downstream: taagccggctgagatcttgt. The upstream and downstream oligonuclotide primers were chosen on both sides of an intron to prevent amplification of possible contaminating gemonmic DNA. mRNA quantities were normalized against $18 \mathrm{~s}$ as an endogenous control for each sample.

\section{Immunoblot analysis of mammary tissue}

Frozen mammary glands were homogenized in protein lysis buffer to extract whole proteins as described [11]. Protein concentration was quantified using the bicinchoninic protein assay (Pierce, Rockford, IL, USA). Protein $(50 \mu \mathrm{g})$ from each sample was solubilized in sample dilution buffer, separated by denaturing 4-15\% NuPage gels (Invitrogen Life Technologies, Carlsbad, CA, USA) and electrophoretically transferred to polyvinylidene difluoride membranes for immunoblot analysis. Membranes were blocked in 5\% nonfat dry milk in Tris buffered saline and $1 \%$ Tween (TBS-T) overnight at $4^{\circ} \mathrm{C}$. Membranes were incubated with a 1:1000 dilution of primary antibody against rat monoclonal to CD31 (ab28364,Abcam, Cambridge, MA), for $1 \mathrm{hr}$ at room temperature and washed with TBS-T. Bound antibody was detected by incubation with a 1:5000 dilution of a horseradish peroxidase-linked anti-rat IgG secondary antibody (A9037, Sigma, St. Louis, MO) for $1 \mathrm{hr}$ at room temperature. After washing with TBS-T, immunoreactive protein was detected with the enhanced chemiluminescence (ECL) plus Western blotting detection kit (Amersham Biosciences, Piscataway, NJ, USA).

\section{Statistical analysis}

Statistical differences among groups were analyzed using $t$ tests using GraphPad Prism (GraphPad Software, San Diego, CA). Data are presented as means \pm S.E.M. Significance was assigned at $P \leq 0.05$.

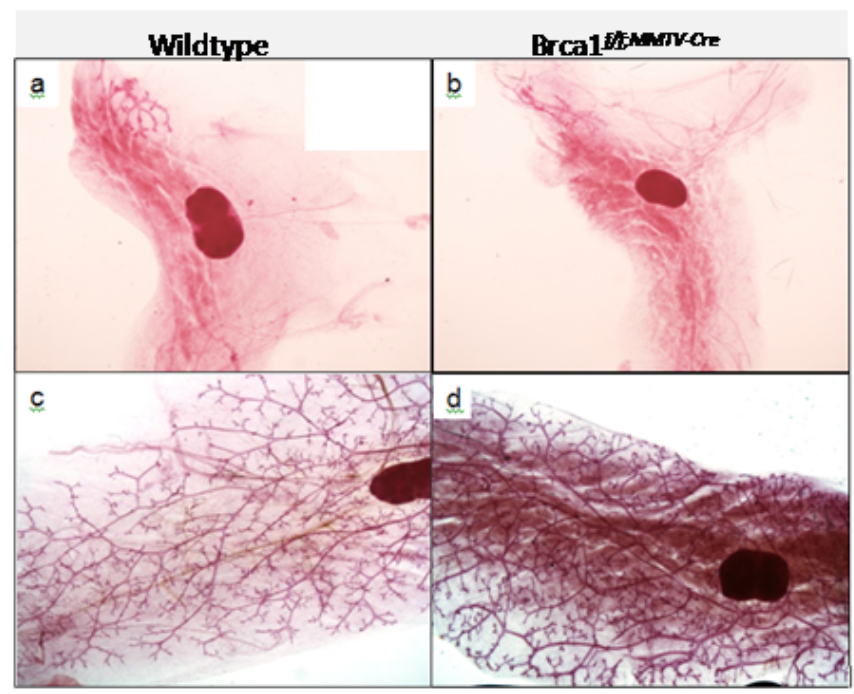

Figure 1: Brca1 mutant mice exhibit an abnormally persistent dense mammary fat pad into adulthood. Representative mammary gland whole mounts of wildtype (a) and Brca $1^{\text {ff/;/MMTV-Cre }}$ (b) mice at 3 weeks of age and at 6 months of age $(\mathbf{c}, \mathbf{d})$, respectively. Magnification, $\times 1$. 


\section{Results}

Representative mammary glands from 3 week old mice demonstrate the presence of both WAT and BAT in the mammary gland of wildtype (Figure 1a) and Brcal mutant mice (Figure 1b). After puberty, adult wildtype mice normally lose the BAT phenotype (Figure 1c). In contrast, we observed a markedly dense area in the mammary fat pad of all the Brcal mutant mice analyzed (Figure 1d). As expected, wildtype mice histologically displayed predominantly white adipocytes with large, unilocular morphology (Figure 2a). In contrast, we observed that the dense area in the mammary fat pad of Brcal mutant mice had smaller, multilocular adipocytes that are histologically reminiscent of BAT (Figure $2 \mathrm{~b}$ ). We found this multilocular BAT phenotype to be unique to the mammary fat pads of Brcal mutant mice since we did not observe this phenotype in other adult wildtype mouse models examined (data not shown). Using a marker for BAT, UCP1, we demonstrated that while the mammary adipose in wildtype mice was negative for the UCP1 antibody staining (Figure 2c), Brcal mutant mice showed positive staining in the same regions within the mammary fat that looked morphologically like BAT (Figure 2d). We also quantified UCP1 at the transcription level. Levels of a housekeeping gene (18s) were measured as an internal standard, and results were normalized to the level of the age-matched wiltype mice in each case. We observed that the expression of UCP1 mRNA in the Brcal mutant mice was significantly greater ( $\sim 50$-fold) than over levels observed in the agematched mammary glands from wildtype mice (data not shown).

Notably, BAT has characteristics that are also favorable for tumor growth, including increased vascularity. To examine whether the multilocular BAT phenotype in the mammary fat pad had characteristics of increased vascularity, we stained the mammary tissue with CD31, a marker commonly used to measure angiogenesis. We found that the regions of mammary fat pad in Brcal mutant mice that was histologically reminiscent of multilocular BAT, also stained positive

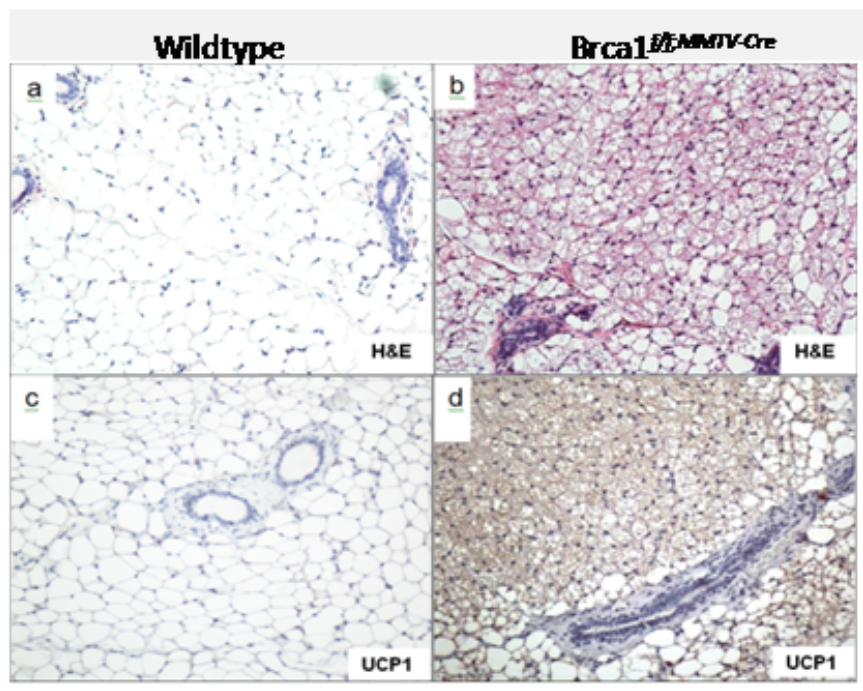

Figure 2: Dense staining on Brca1 mutant mouse wholemount morphologically resembles multilocular brown adipose and stains positive for UCP1. $\mathrm{H} \& \mathrm{E}$ analysis showing the mammary fat pad of wildtype (a) and Brca1 $1_{\mathrm{ffF} ; \mathrm{MMTV}-\mathrm{Cre}}$ (b) postpubertal mice (6 months of age). Immunohistochemical detection of the brown adipose tissue marker, UCP1 in the mammary fat pad of wildtype (c) and Brca1 $1^{\text {ff;:MMTV-Cre }}$ (d) mice. Magnification, x 20.

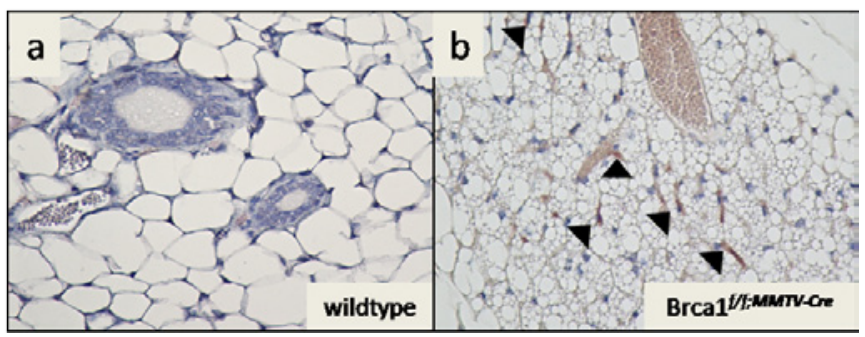

Figure 3: Multilocular brown adipose appearance in Brca1 mutant mice shows increased staining for CD31, a marker of angiogenesis. Immunohistochemical detection of the angiogenesis marker, CD31 in the mammary fat pad of wildtype (a) and Brca $1^{\mathrm{fff} \text {;MMTV-Cre }}$ (b) postpubertal mice (6 months of age). Magnification, $x 40$. Arrowheads point to areas with CD31 positive staining.

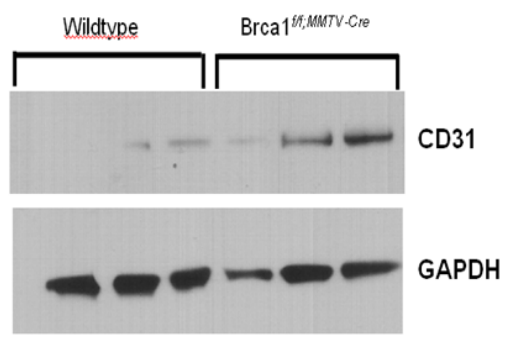

Figure 4: Immunoblot analysis demonstrates that CD31 protein is increased in Brca1 mutant mouse mammary gland. Representative protein lysates from mammary glands from Brca1 mutant and wildtype mice were subjected to Western blotting (as described in materials and methods) to detect CD31 and GAPDH (control for loading and transfer).

for CD31 (Figure 3b) in contrast to wildtype mice which showed no staining for CD31 (Figure 3a). We also observed that the protein expression of CD31 in the Brcal mutant mouse mammary glands were 2 -fold greater than levels seen in the age-matched mammary glands from wildtype mice (Figure 4).

\section{Discussion}

This study reports the unexpected sustained deposition of multilocular adipocytes in the adult mammary gland of Brcal mutant mice which was not seen in other wildtype mouse models. Additionally, the BAT phenotype was correlated with increased angiogenesis. Indeed it is known that angiogenesis is an essential step for breast cancer progression and dissemination [20]. Given that adipose tissue is the major contributor to the volume of the breast, these findings raise questions as to whether the sustained presence of multilocular brown adipocytes in mammary adipose tissue can provide a permissive environment for tumor growth.

The mammary gland is a unique organ which continually undergoes changes in growth and its architecture from birth to the adult [1-4] After birth, a rudimentary ductal tree remains quiescent in the gland until a robust proliferative growth and branching begins at the onset of puberty [1-4]. This stage of rapid mammary ductal growth is carefully orchestrated and controlled by key hormones and growth factor signals (e.g. estrogen and Insulin like growth factor-1 (IGF-1) that extend the ductal tree [1-4]. Key structures, called terminal end buds (TEBs), are highly proliferative formations at the tips of the ducts that drive the ducts to penetrate farther into the fat pad until the end of puberty [14]. At the end of puberty when ductal outgrowth is complete, the TEBs 
normally differentiate into terminal ducts that remain quiescent until additional signals are received to induce a proliferative response (e.g. estrous cycling, pregnancy) [1-4]. The completion of ductal outgrowth normally coincides with the disappearance of BAT in the mammary fat pad $[8,9]$. Thus, it is not clear why the BAT phenotype abnormally persists in the mammary gland Brcal mutant mice well into adulthood. Although, the anatomical location of depots of WAT and BAT is relatively distinct, studies have shown that adipose tissue has a degree of plasticity and can switch from WAT to BAT and vice-versa in response to certain endogenous and exogenous stimuli [21,22]. In the case of mammary adipose, the BAT phenotype is normally detected during puberty which is also a time when high levels of hormones and growth factors are also present $[8,9]$. Although not investigated, it is possible that the transient deposition of BAT in the pubertal mammary gland could be the result of a systemic effect driven by hormone and growth factor signaling (e.g. estrogen and IGF-1). This would explain why the disappearance of these factors after puberty leads to a mammary fat pad primarily composed of unilocular adipocytes [8]. Evidence supporting this hypothesis are experimental studies from Matsumoto et al. $[23,24]$ who demonstrated in ovariectomized mice that estrogen and progesterone treatments can alter the deposition of unilocular and multilocular adipocytes in the mammary fat pad. Additionally, other studies demonstrated that estrogen deficiency in ovariectomized rats is followed by reduced UCP1 expression [25] while other studies have demonstrated that IGF-1 can induce UCP-1 expression in vitro and in vivo models [26,27]. Indeed, several lines of evidence have demonstrated that both experimental mouse models and humans that are deficient in BRCA1 have abnormally high levels of estrogen/IGF-1 signaling and production [28-33]. In particular, experimental and epidemiological studies have demonstrated that loss of BRCA1 function can lead to the loss of the normal restraint on estrogen receptor signaling [34-36] and also an increase in estrogen production through increased expression of aromatase, an enzyme responsible for a key step in the biosynthesis of estrogens [37]. Also, prior studies in the same mouse model used in these studies have revealed that the absence of Brcal results in increased expression of several components of the IGF-1 axis in the liver, normal mammary tissue, and mammary tumors and increased levels of serum IGF-1 [28,38]. Therefore, if high levels of estrogen/IGF-1 signaling and production can alter the mammary stromal microenvironment to a phenotype that provides a permissive environment for tumor cell growth, this would exacerbate the known synergistic effects of estrogen/ IGF-1 to drive mammary epithelial cell growth. We are pursuing more mechanistic studies to determine whether the known characteristics of abnormal hormonal/growth factor signaling in a Brcal deficient setting drives the persistent BAT phenotype in mammary adipose.

Finally, the unexpected finding of the BAT phenotype in the mammary gland of Brcal mutant mice raises an important issue regarding whether the BAT phenotype can also be detected in humans, particularly those with BRCA1 mutations. It has long been assumed that adults do not possess more than vestigial amounts of BAT due to the general contention that BAT is gradually lost postnatally when its specific role in adaptive thermogenesis is normally concluded [6,7]. However, recent morphological and imaging studies demonstrate that BAT is present in adults and can also be stimulated to appear in traditional WAT depots $[6,7,39-42]$. To date, there is only limited understanding of the distribution of WAT and BAT within the normal human breast. Multilocular adipocytes have been detected in the human infant mammary gland $[43,44]$. In particular, analysis of ductal structures from human neonates have demonstrated that these structure are often embedded within a connective tissue stroma and also in close proximity with nearby islands of multilocular, vascularized embryonic fat $[43,44]$. Additionally, there are several reports that have documented the observation of brown fat (hibernomas) in breast tumors [45-51]. Whether BRCA1 mutation carriers possess more BAT compared to non-BRCA1 mutations carriers remains to be determined. Notably, BAT is inversely correlated with obesity and body mass index (BMI) $[52,53]$. One study reporting that women with an inherited high risk of breast cancer often present as young and slim patients with a low BMI [54] supports the notion that women with inherited BRCA1 mutations may actually possess more BAT compared to those patients without mutations in Brca1. Although, a long-standing line of evidence suggests that mammary adipose is essential for ductal morphogenesis and breast cancer [55-56], one important consideration to take into account is the fact that there are fundamental differences in the microenvironment of the adult rodent and human mammary glands [55]. In particular, the rodent microenvironment is comprised primarily of an adipose stroma with epithelial parenchyma versus the human gland, which is composed of a fibrous-adipose stroma with epithelial parenchyma [55] Taking this into consideration, it is likely that the sustained presence of multilocular brown adipocytes would be most relevant for invasive tumors that break through the basement membrane resulting in an immediate juxtaposition of adipocytes and breast cancer cells allowing paracrine interactions between the two cell types. Thus, a persistent BAT phenotype in mammary adipose could indeed provide a permissive environment for tumor growth. Our findings provide a framework for which to pursue mechanistic investigations regarding if/how the plasticity of adipose tissue can impact breast cancer development in the adult. This Brca1 mutant mouse model should provide a physiologically relevant context for these investigations.

\section{Acknowledgements}

This work was supported by the $\mathrm{NIH}$, National Cancer Institute Grant CA062483-28S1 and in part by the Mid-Atlantic Nutrition Obesity Research Center (NORC) - NIH Grant P30DK072488.

\section{References}

1. Beckmann MW, Niederacher D, Schnürch HG, Gusterson BA, Bender HG (1997) Multistep carcinogenesis of breast cancer and tumour heterogeneity. $J$ Mol Med 75: 429-439.

2. Russo IH, Russo J (1998) Role of hormones in mammary cancer initiation and progression. J Mammary Gland Biol Neoplasia 3: 49-61.

3. Fendrick JL, Raafat AM, Haslam S (1998) Mammary gland growth and development from the postnatal period to postmenopause: ovarian steroid receptor ontogeny and regulation in the mouse. J Mammary Gland Bio Neoplasia 3: 7-22.

4. Robinson GW, Karpf AB, Kratochwil K (1999) Regulation of mammary gland development by tissue interaction. J Mammary Gland Biol Neoplasia 4: 9-19.

5. Bissell MJ, Hines W (2011) Why don't we get more cancer? A proposed role of the microenvironment in restraining cancer progression. Nat Med 17: 320-329.

6. Cannon B, Nedergaard J (2004) Brown adipose tissue: function and physiological significance. Physiol Rev 84: 277-359.

7. Cannon B, Nedergaard J (2008) Studies of thermogenesis and mitochondrial function in adipose tissues. Methods Mol Biol 456: 109-121.

8. Gouon-Evans V, Pollard J (2002) Unexpected deposition of brown fat in mammary gland during postnatal development. Mol Endocrinol 16: 2618-2627.

9. Master SR, Hartman JL, D'Cruz CM, Moody SE, Keiper EA, et al. (2002) 
Functional microarray analysis of mammary organogenesis reveals a developmental role in adaptive thermogenesis. Mol Endocrinol 16: 1185-1203.

10. Walden PD, Ruan W, Feldman M, Kleinberg DL (1998) Evidence that the mammary fat pad mediates the action of growth hormone in mammary gland development. Endocrinology 139: 659-662.

11. Jones LP, Li M, Halama ED, Ma Y, Lubet R, et al. (2005) Promotion of mammary cancer development by tamoxifen in a mouse model of Brca1-mutation related breast cancer. Oncogene 24: 3554-3562.

12. Jones LP, Tilli MT, Assefnia S, Torre K, Halama ED, et al. (2008) Activation of estrogen signaling pathways collaborates with loss of Brca1 to promote development of ERalphạ-negative and ERalpha-positive mammary preneoplasia and cancer. Oncogene 27: 794-802.

13. Jones LP, Sampson A, Kang HJ, Kim HJ, Yi YW, et al. (2010) Loss of BRCA1 leads to an increased sensitivity to Bisphenol A. Toxicology Lett 199: 261-268.

14. Ford D, Easton DF, Stratton M, Narod S, Goldgar D, et al. (1998) Genetic heterogeneity and penetrance analysis of the BRCA1 and BRCA2 genes in breast cancer families. The Breast Cancer Linkage Consortium. Am J Hum Genet 62: 676- 689.

15. Xu X, Wagner KU, Larson D, Weaver Z, Li C , et al. (1999) Conditional mutation of Brca1 in mammary epithelial cells results in blunted ductal morphogenesis and tumour formation. Nat Genet 22: 37-43.

16. Weaver Z, Montagna C, Xu X, Howard T, Gadina M, et al. (2002) Mammary tumors in mice conditionally mutant for Brca1 exhibit gross genomic instability and centrosome amplification yet display a recurring distribution of genomic imbalances that is similar to human breast cancer. Oncogene 21: 5097-5107.

17. Frech MS, Jones LP, Furth PA (2006) Validation of transgenic models of breast cancer: Ductal Carcinoma In Situ (DCIS) and Brca1 mutation related breast cancer. Breast Cancer Online 8: e42.

18. Herschkowitx JI, Simin K, Weigman VJ, Mikaelian I, Usary J, et al. (2007) Identification of conserved gene expression features between murine mammary carcinoma models and human breast tumors. Genome Biology 8: R76.

19. Wagner KU, McAllister K, Ward T, Davis B, Wiseman R, et al. (2001) Spatia and temporal expression of the Cre gene under the control of the MMTV-LTR in different lines of transgenic mice. Transgenic Res 10: 545-553.

20. Longatto Filho A, Lopes JM, Schmitt FC (2010) Angiogenesis and breas cancer. J Oncol pii: 576384

21. Seale P, Kajimura S, Spiegelman BM (2009) Transcriptional control of brown adipocyte development and physiological function--of mice and men. Genes Dev 23: 788-797.

22. Frontini A, Cinti S (2010) Distribution and development of brown adipocytes in the murine and human adipose organ. Cell Metab 11: 253-256.

23. Matsumoto M, Nishinakagawa H, Kurohmaru M, Hayashi $Y$ (1995) Effects of estrogen and progesterone on the parenchyma and blood vessels of the mammary gland in ovariectomized adult mice. J Vet Med Sci 57: 39-44.

24. Matsumoto M, Nishinakagawa H, Kurohmaru M, Hayashi Y, Otsuka J (1992) Effects of estrogen and progesterone on the development of the mammary gland and the associated blood vessels in ovariectomized mice. J Vet Med Sci 54: 1117-1124

25. Pedersen SB, Bruun JM, Kristensen K, Richelsen B (2001) Regulation of UCP1, UCP2, and UCP3 mRNA expression in brown adipose tissue, white adipose tissue, and skeletal muscle in rats by estrogen. Biochem Biophys Res Commun 288: 191-197.

26. Silva JE, Rabelo R (1997) Regulation of the uncoupling protein gene expression. Eur J Endocrinol 136: 251-264.

27. Duchamp C, Burton KA, Géloën A, Dauncey MJ (1997) Transient upregulation of IGF-I gene expression in brown adipose tissue of cold-exposed rats. Am J Physiol 272: E453-E460.

28. Shukla V, Coumoul X, Cao L, Wang RH, Xiao C, et al. (2006) Absence of the Full-Length Breast Cancer-Associated Gene-1 Leads to Increased Expression of Insulin-Like Growth Factor Signaling Axis Members. Cancer Res 66: 71517157.
29. Shukla V, Coumoul X, Vassilopoulos A, Deng CX (2008) IGF signaling pathway as a selective target of familial breast cancer therapy. Curr Mol Med 8: 727-740.

30. Hudelist G, Wagner T, Rosner M, Fink-Retter A, Gschwantler-Kaulich D, et al. (2007) Intratumoral IGF-I protein expression is selectively upregulated in breast cancer patients with BRCA1/2 mutations. Endocr Relat Cancer 14: 1053-1062.

31. Maor S, Yosepovich A, Papa MZ, Yarden RI, Mayer D, et al. (2007) Elevated insulin-like growth factor-I receptor (IGF-IR) levels in primary breast tumors associated with BRCA1 mutations. Cancer Lett 257: 236-243.

32. Rosen EM, Fan S, Isaacs C (2005) BRCA1 in hormonal carcinogenesis: basic and clinical research. Endocr Relat Cancer 12: 533-548.

33. Berstein LM (2008) Endocrinology of the wild and mutant BRCA1 gene and types of hormonal carcinogenesis. Future Oncol 4: 23-39.

34. Fan S, Wang J, Yuan R, Ma Y, Meng Q, et al. (1999) BRCA1 inhibition of estrogen receptor signaling in transfected cells. Science 284: 1354-1356.

35. Fan S, Ma YX, Wang C, Yuan RQ, Meng Q, et al. (2002) p300 Modulates the BRCA1 inhibition of estrogen receptor activity. Cancer Res 62: 141-151.

36. Razandi M, Pedram A, Rosen EM, Levin ER (2004) BRCA1 inhibits membrane estrogen and growth factor receptor signaling to cell proliferation in breast cancer. Mol Cell Biol 24: 5900-5913.

37. Ghosh S, Lu Y, Katz A, Hu Y, Li R (2007) Tumor suppressor BRCA1 inhibits a breast cancer-associated promoter of the aromatase gene (CYP19) in human adipose stromal cells. Am J Physiol Endocrinol Metab 292: E246-E252.

38. Jones LP, Stefansson S, Kim MS, Ahn SN (2011) Comparison of radioimmuno and carbon nanotube field-effect transistor assays for measuring insulinlike growth factor-1 in a preclinical model of human breast cancer. J Nanobiotechnology 9: 36

39. Schulz TJ, Huang TL, Tran TT, Zhang H, Townsend KL, et al. (2011) Identification of inducible brown adipocyte progenitors residing in skeletal muscle and white fat. Proc Natl Acad Sci USA 108: 143-148.

40. Truong MT, Erasmus JJ, Munden RF, Marom EM, Sabloff BS, et al. (2004) Focal FDG uptake in mediastinal brown fat mimicking malignancy: a potentia pitfall resolved on PET/CT. AJR Am J Roentgenol 183: 1127-1132.

41. Cook GJ, Fogelman I, Maisey MN (1996) Normal physiological and benign pathological variants of 18-fluoro-2-deoxyglucose positron- emission tomography scanning: potential for error in interpretation. Semin Nucl Med 26 : 308-314

42. Cohade C, Osman M, Pannu HK, Wahl RL (2003) Uptake in supraclavicular area fat ("USA-fat"): description on 18F-FDG PET/CT. J Nucl Med 44: 170-176.

43. Anbazhagan R, Bartek J, Monaghan P, Gusterson BA (1991) Growth and development of the human infant breast. Am J Anat 192: 407-417.

44. Anbazhagan R, Gusterson BA (1995) Ultrastructure and immunohistochemistry of the embryonic type of fat identified in the human infant breast. Anat Rec 241 129-135.

45. Shammas A, Lim R, Charron M (2009) Pediatric FDG PET/CT: physiologic uptake, normal variants, and benign conditions. Radiographics 29: 1467-1486.

46. Kapucuoglu N, Percinel S, Angelone A (2008) Adenohibernoma of the breast Virchows Arch 452: 351-352.

47. Rousseau C, Bourbouloux E, Campion L, Fleury N, Bridji B, et al. (2006) Brown fat in breast cancer patients: analysis of serial 18F-FDG PET/CT scans. European Journal of Nuclear Medicine and Molecular Imaging 33: 785-791.

48. Padilla-Rodriguez AL (2011) Pure hibernoma of the breast: insights about its origins. Ann Diagn Pathol [Epub ahead of print].

49. Colville J, Feigin K, Tang L, Keating D, Cohen MA (2006) Mammary hibernoma Breast J 12: 563-565.

50. Gardner-Thorpe D, Hirschowitz L, Maddox PR (2000) Mammary hibernoma Eur J Surg Oncol 26: 430.

51. Martini N, Londero V, Machin P, Travaini LL, Zuiani C, et al. (2010) An unusua breast lesion: the ultrasonographic, mammographic, MRI and nuclear medicine findings of mammary hibernoma. $\mathrm{Br} \mathrm{J}$ Radiol 83: e1-e4. 
Citation: Jones LP, Buelto D, Tago E, Owusu-Boaitey KE (2011) Abnormal Mammary Adipose Tissue Environment of Brca1 Mutant Mice Show a Persistent Deposition of Highly Vascularized Multilocular Adipocytes. J Cancer Sci Ther S2. doi:10.4172/1948-5956.S2-004

Page 6 of 6

52. Cypess AM, Kahn CR (2010) Brown fat as a therapy for obesity and diabetes. Curr Opin Endocrinol Diabetes Obes 2: 143-149.

53. Frühbeck G, Becerril S, Sáinz N, Garrastachu P, García-Velloso MJ (2009) BAT: a new target for human obesity? Trends Pharmacol 30: 387-396.

54. Werdin F, Peek A, Martin NC, Baumeister S (2010) Superior gluteal artery perforator flap in bilateral breast reconstruction. Ann Plast Surg 64: 17-21.
55. Hovey RC, McFadden TB, Akers RM (1999) Regulation of mammary gland growth and morphogenesis by the mammary fat pad: a species comparison. $J$ Mammary Gland Biol Neoplasia 4: 53-68.

56. Neville MC, Medina D, Monks J, Hovey RC (1998) The mammary fat pad. J Mammary Gland Biol Neoplasia 3: 109-116. 